\title{
Glanceability Evaluation of a Physical Activity Feedback System for Office Workers
}

\author{
S.T. Boerema ${ }^{1,2}$, R. Klaassen ${ }^{3}$, H.J.A. op den Akker $^{3}$, H.J. Hermens ${ }^{1,2}$ \\ ${ }^{1}$ Roessingh Research and Development, Roessinghsbleekweg 33b, Enschede, the Netherlands \\ ${ }^{2}$ Telemedicine, University of Twente, P.O. Box 2177500 AE, Enschede, the Netherlands \\ ${ }^{3}$ Human Media Interaction, University of Twente, P.O. Box 2177500 AE, Enschede, the Netherlands \\ s.boerema@rrd.nl, r.klaassen@utwente.nl,.h.j.a.opdenakker@utwente.nl, h.hermens@rrd.nl
}

Keywords: Glanceability, Feedback, Physical Activity, User-centered Design, ICT Applications, Behaviour Change Support System.

\begin{abstract}
This paper presents the results of a user evaluation to design a glanceable user interface presenting physical activity feedback to office workers during the workday. The feedback is presented on a central and public display next to the coffee machine in the office building. Users should be able to receive the feedback quickly and easily while getting a cup of coffee - the user interface should be glanceable. The feedback should communicate the (real-time) amount of physical activity and the progress toward the goal of the user for a day. Three mock-ups of user interfaces were developed and evaluated in a user evaluation study. Differences on reaction time and user preferences were found. None of these results were significant. Adding group information to the mock-up increases reaction time and therefore lowers the glanceability negatively.
\end{abstract}

\section{INTRODUCTION}

Not being sufficiently physically active is regarded as one of the most important independent risk factors of reduced life expectancy. It increases the risk of obesity, coronary heart disease and stroke, type 2 diabetes, as well as colon and breast cancer. It is therefore recognised as one of the most important modifiable risk factors that is causing the rising global burden of chronic disease.

About $40 \%$ of Dutch adults are not sufficiently physically active and over $40 \%$ are overweight or obese. Studies showed pooled prevalence of sedentary lifestyles for 15 European countries being $31 \%$, whereas $17.7 \%$ of the population of 51 mainly low- and middle-income worldwide countries were physically inactive, indicating that inactivity may be more prevalent in wealthier countries (Guthold et al. 2008). Many contemporary work tasks are characterised by little or no physical activity. More than a quarter of all employees in the Netherlands have sedentary work and sit on average 4 hours while being at work and travelling to and from work. TNO Care and Prevention (Hildebrandt n.d.) states that, in the Netherlands, employees that participate in sports report less ill and mostly for a shorter period then their non-sporting colleagues. This effect is most strongly for employees with sedentary work. Given the various health benefits of physical activity, and the high prevalence of physical inactivity during work, many health interventions focus on promoting physical activity.

Providing feedback on the personal physical activity level can create awareness and motivation to change physical behaviour, as described in many social cognitive models on health behaviour (Nutbeam \& Harris 2004). Physical activity interventions focussed on sedentary workers have been using self reporting methods (e.g. questionnaires) and pedometers (i.e. step-counters) (Chan et al. 2004; Dinger et al. 2007; Cocker et al. 2008). These studies provided feedback on an individual level, not incorporating direct interference of the social environment, while social norms are often considered an important factor in behaviour change, as described in the Health Belief Model, the Social Cognitive Theory and the Theory of Planned 
Behaviour (Rosenstock 1966; Bandura et al. 1977; Ajzen 1991).

The office setting is an interesting environment for studying group mechanisms in physical activity feedback on the self-awareness and behaviour of sedentary workers. To study these group mechanisms, a system will be developed based on wearable physical activity sensors and a feedback device. We envision that the feedback device will be used by multiple users in a public space of the office containing personal and group feedback. This vision implies high demands on the usability and understanding of the graphical user interface (GUI) of the feedback device. The goal of the system is to create awareness and motivation people to be more physical active, which can result in a healthier lifestyle at the office. In this system, physical activity is measured by a hip mounted activity monitor - which can estimate energy expenditure based on a 3D accelerometer according to the method of Bouten (1996) - and this data is wireless and real-time transmitted to and processed on a central server. Based on the data the system presents feedback on the central display of the system, which is located near the coffee machine in the office building. While waiting for their cup of coffee the system shows real-time feedback about the physical activity until that moment of the current workday. The goal of this research is to design a user interface to present feedback to the users on the central display. Because of its setting at the coffee machine the feedback interpretation time is limited. The designed user interface should therefore be glanceable. Matthews defines glanceable as follows: "By glanceable, we mean enabling users to understand information quickly and easily. Glanceability is critical to peripheral display design because users need to quickly glance at and read displayed information with minimal interruption to their primary task" (Matthews 2006; Matthews et al. 2007).

The effect on glanceability of various user interfaces and the addition of group information to personal feedback will be studied during a user evaluation, in which reaction time will be used as a measure for 'quickly' and the correctness of the interpretation of the information in the mock-up as a measurement for 'easily'. From the results of the user evaluation we conclude which of the designed user interface is the most glanceable, what is the effect of adding group information to user interface on the glanceability, and which user interface is preferred by the subjects.

\section{METHODOLOGY}

Three mock-ups of the envisioned physical activity feedback system were compared in a controlled user experiment to answer the research questions. To measure the effect of glanceability the mock-ups were compared using a within-subject design while the effect of adding group information to the mockups was compared in a between-subject design.

Personal information and information about sitting behaviour, workday activities, physical activity and sport are gathered at the beginning of the experiment.

During the actual user experiment the participant will evaluate the three mock-ups to measure the clarity and glanceability of the mock-ups. Two questions were given with each mock-up: one about the amount of physical activity and one about the progress towards their goal. The questions were the same for all participants. Participants in the "with group information' group had to answer a third question about their own performance compared to the group performance. The participants can answer the question on a five point Likert scale (1 for very bad, to 5 for very good) and were asked to: "answer the question correctly and as quickly as possible". The correctness of the interpretation is used to calculate the clarity of the mock-up (whether the answer is correct or not), while the reaction time (the time the participant needs to answer the question) is used to calculate the glanceability of the mock-up. Incorrect answers were excluded from reaction time calculations.

After each mock-up participants evaluated usability and their perception of information on the screen. This questionnaire was adapted from Quesenbery (2003; Stone e.a. 2005) and was extended by two questions on their intention to use the system in future and the attractiveness of the system.

At the end of the experiment the participants were invited for a short, semi-structured interview about their preference of one of the mock-ups and their opinion on the general idea of presenting feedback on physical activity on a public screen in an office environment.

The results of the reaction times and the answers to the questionnaire of the three mock-ups were compared using a mixed between-within ANOVA test. The results of the questionnaires were recorded and interesting remarks will be presented in the result section. 


\subsection{Subjects}

Subjects were recruited from two offices by the snowball sampling method. The only inclusion criterion was: doing mainly deskwork.

Participants were randomly assigned to one of two conditions: 1) presenting three mock-ups without the group comparison ('no group') and 2) presenting three mock-ups with the group comparison ('group').

\subsection{Procedure}

The subjects were invited to participate in the experiment. When the subjects agreed taking part in the experiment they received a short written introduction on physical activity and deskwork. The introduction included a scenario in which the envisioned system was introduced in an office setting.

The order of presenting the three mock-ups was counterbalanced. During the user evaluations the participants were observed, notes were taken and audio recordings of the interviews were stored. Filling in the questionnaire and evaluating the mockups were done on separate computers.

\subsection{Design of Mock-ups}

For both conditions three different mock-ups are a Number, a Flower and a Graph. All user interfaces present real-time physical activity data and the progress of reaching the goal. In this the progress is given in minutes of physical activity up to the moment of walking by the feedback device and the goal being a total of 30 minutes of physical activity during office hours.

The first mock-up uses a number to present the progress reaching the goal in a percentage (for example $73 \%$ ). The number presents the progress towards the goal of the user. The background colour presents how the user is distributing the amount of activity over the day. Group information is added to the mock-up by text stating the average score of the group and the number of people that already reached their goal.

The second mock-up uses a metaphor of a growing flower to present the progress in physical activity. The stage of the flower represents the progress towards the goal of the user. The exact percentage towards to the goal of the user is displayed in the heart of the flower. Group information is added to the mock-up by the metaphor of a garden. Every flower represents a user of the system. The garden provides an overview of all the activities of all users.

The third mock-up presents physical activity of the user in a graph. This mock-up is an improved mock-up of the system and is based on the work of Boerema (2009). The graph provides an overview of the amount of physical activity per hour. Group information is shown in the mock-up by a line that shows the average level of physical activity of the group. The six mock-ups are given in Figure 1.

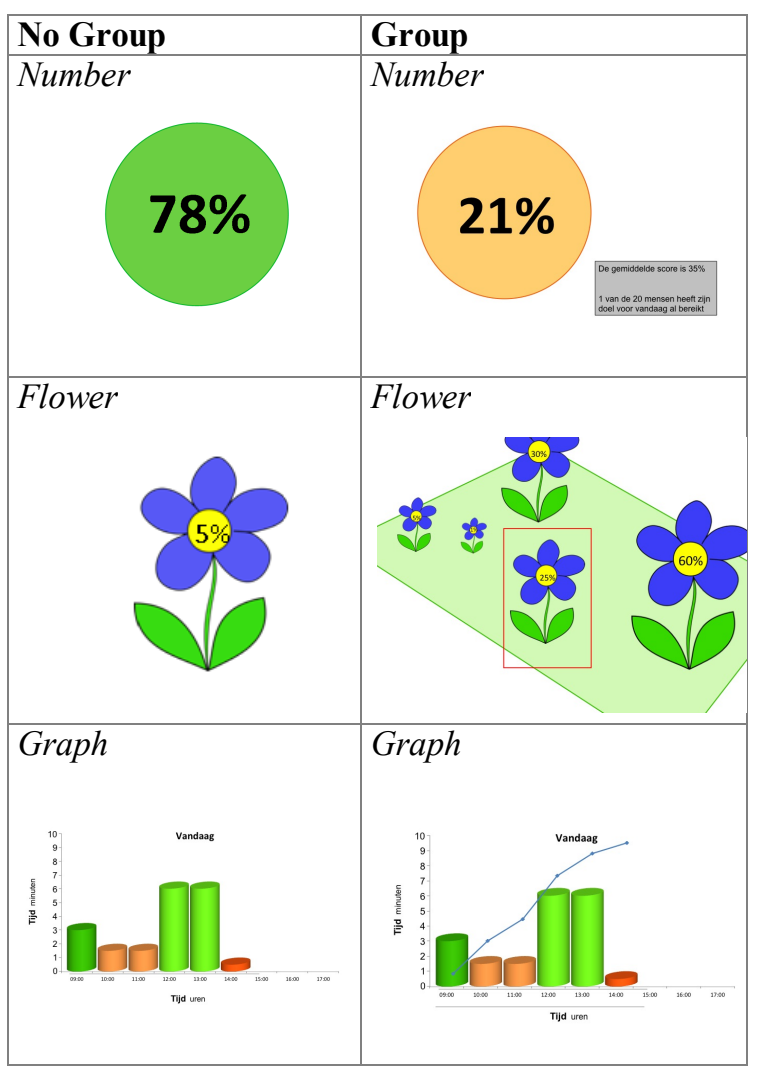

Figure 1: Overview of the six mock-ups.

\section{RESULTS}

\subsection{Subjects}

Twenty seven people participated in the experiment. From this, four were excluded from the data analysis because they did not understand the experimental setup, and expressed this during the experiment. From the remaining 23 subjects, 12 were male and 11 female, with an average age of $31 \pm 7$ years.

The participants were all office workers (researchers, administrative staff or undergraduate students). They reported to have an average working 
day of $8.1 \pm 0.5$ hours on which they spend on average $7.1 \pm 1.0$ hours, sitting. On the question whether they engage in sports, 14 subjects responded positive, 3 negative, and 6 answered 'rarely', On the question whether the subject considers himself or herself physically active above the average, the response on a 5 point Likert scale: not agree - agree, was $3 \pm 1.2$, which means that they were neutral about their physical activity level compared to others.

Twelve participants were assigned to the mockups without the group comparison and 11 participants were assigned to the mock-ups with the group comparison.

\subsection{Intention to Use}

After reading the scenario, participants rated their intention to use the system, on a Likert scale 1-5: negative - positive. They average response was 3.4 \pm 1.0 , meaning that they were slightly positive on using the system. There was no correlation between the self reported engagement in sports, the physical activity level and intention to use.

After each mock-up, intention to use was asked again. Randomization of the mock-ups was tested by studying order effects in intention to use. There was no trend in intention to use answers neither towards the positive nor the negative, therefore answers per mock-up can be compared without correcting for the order in which they were presented to the subject.

In both the 'no group' and 'group' conditions comparison the intention to use was the lowest after seeing the 'Graph': $2.5 \pm 1.3$. Intention to use after 'Number' was on average $3.3 \pm 1.0$ and after 'Flower', $2.8 \pm 1.2$. Only in the No Group condition the intention to use after Flower was below 3, being neutral. Results per condition are shown in Figure 2.

\subsection{Correctness}

In the 'no group' condition only one subject answered 'I don't know' while using the Graph mock-up. In the 'Group' condition multiple subjects answered 'I don't know', three subjects while using the Number mock-up, three subjects while using the Flower mock-up and one subject while using the Graph mock-up.

All information perception questions were correctly answered, except for the 'no group' Flower condition, which was mostly answered as showing sufficient physical activity (while the flower displayed a lower level of physical activity) and a

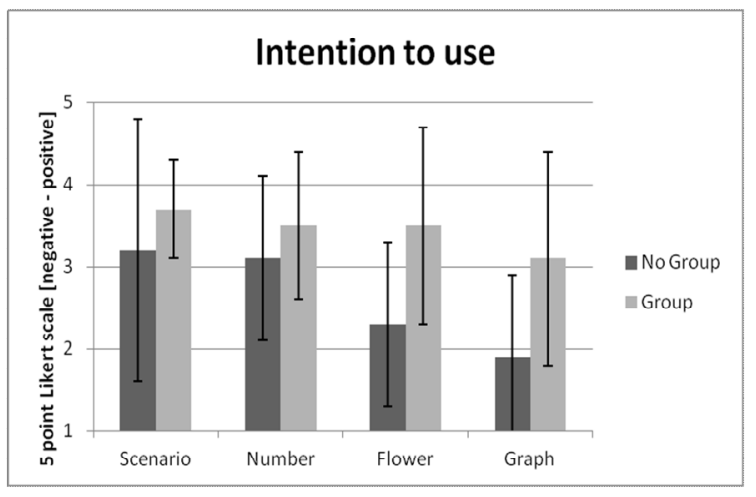

Figure 2: Intention to use the system per condition ('No Group' and 'Group'), self reported on a 5 point Likert scale: negative - positive. Self reports are given after reading the scenario and after seeing each mock-up.

good progress towards the goal (while the flowers displayed a bad progress towards their goal).

\subsection{Reaction Time}

The randomisation of the mock-ups was also tested by studying order effect on reaction time. There was no order effect in reaction time for the questions, indicating that reaction times can be compared without correcting for the order in which subjects have seen the mock-ups.

In figure 3 and 4 are the average reaction times given per group, per mock-up. The reaction time on question $2(\mathrm{Q} 2)$ is in all cases much shorter than on question 1 (Q1). For the first question the reaction time is shorter for the No Group condition.

\subsection{Interviews}

At the end of the user evaluation the participants indicated their preference for the mock-ups. Ten

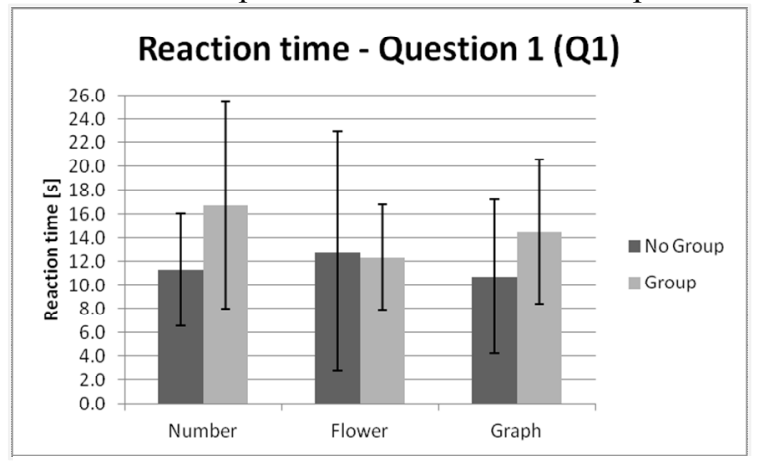

Figure 3: Average reaction time and standard deviation of question 1 about "sufficient physical activity", given per condition. 


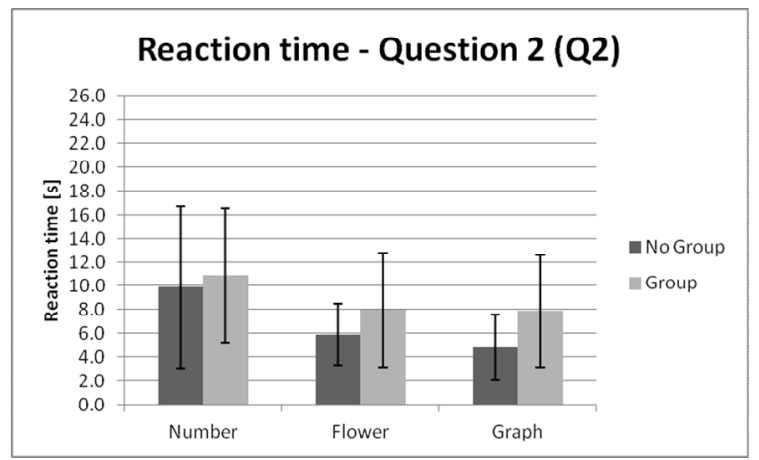

Figure 4: Average reaction time and standard deviation of question 2 about "reaching the personal goal", given per condition.

participants preferred the Number, eight participants preferred the Flower and five participants preferred the Graph. The participants were asked to state their opinion about presenting feedback in a public and central place. Fifteen participants indicated that it was no problem to show their physical activity level on a central and public display. Eight participants indicated that they have issues with the idea of public and central feedback. These participants expressed privacy concerns or were not interested at all in these kinds of feedback systems. Nineteen participants indicated that they would join a system like this in their office, if it would be introduced.

\section{DISCUSSION \& CONCLUSION}

The results of the user evaluation showed no significant differences between the reaction times of the different mock-ups. The Number and Graph showed the shortest response time, see figure 3 and 4. These differences can be explained from the design of the mock-ups. Presenting progress towards a goal by displaying a percentage is one of the simplest ways. Following the definition of glanceability this should be the most glanceable user interface. The Graph showed more details about the amount of physical activity. It can take longer to process the more detailed information. Using a flower as a metaphor for displaying the amount of physical activity is received as joyful, but less intuitive for displaying the amount of physical activity.

No significant differences were found between the two conditions of 'no group' and 'group' feedback. The 'no group' condition showed a shorter reaction time for all the participants on the first two questions. This difference can be explained by the extra time the user needs to interpret the extra information of the group. Group information does affect the correctness of the answer to the question during the user evaluation. In the 'group' condition only one participant was not able to answer a question, while seven participants of the 'group' condition were not able to answer one of the questions.

The results of interviews showed that the participants preferred the 'number' mock-up. The number is also most glanceable mock-up.

A small group of participants showed privacy concerns of displaying public and central feedback on a large screen in an office setting.

When designing a glanceable user interface for the system, it should be taken into account if group information is necessary to influence the user.

\section{FUTURE WORK}

A next step in designing a user interface for the system is the personification of the user interface by adding an embodied conversation (ECA) agent to the user interface of the system. The results from previous studies indicate that the use of an ECA can have a positive effect on how the feedback is received by the user in behaviour change support systems. This can eventually lead to a better performance of the coaching program in the future and a more effective way to support users to change their behaviour (Schulman \& Bickmore 2009; Blanson Henkemans et al. 2009; Berry et al. 2005). The results of this user evaluation and the effect of adding an ECA to the UI are subject of future user evaluations with a working prototype of the system.

\section{ACKNOWLEDGEMENTS}

The authors would like to thank all subjects for participating in this study.

The work of R. Klaassen en H. op den Akker was funded by the European Commission, within the framework of the ARTEMIS JU SP8 SMARCOS project 100249 - (www.smarcos-project.eu).

The work of S. Boerema and H. Hermens was funded by the SENIOR project, within the program of economical innovation 'Pieken in de Delta OostNederland', The Netherlands. 


\section{REFERENCES}

Ajzen, I., 1991. The theory of planned behavior. Organizational Behavior and Human Decision Processes, 50(2), pp.179-211.

Bandura, A., Adams, N. \& Beyer, J., 1977. Cognitiveprocesses mediating behavioral change. Journal of Personality and Social Psychology, 35(3), pp.125139.

Berry, D.C., Butler, L.T. \& De Rosis, F., 2005. Evaluating a realistic agent in an advice-giving task. International Journal of Human Computer Studies, 63(3), pp.304327.

Blanson Henkemans, O.A. et al., 2009. An online lifestyle diary with a persuasive computer assistant providing feedback on self-management. Technology and Health Care, 17(3), pp.253-267.

Boerema, S.T., 2009. Centralised physical activity feedback; encouraging employees working in an office setting to be sufficiently physically active. Master Thesis. Enschede, The Netherlands: Twente University.

Bouten, C.V.C. et al., 1996. Daily physical activity assessment: Comparison between movement registration and doubly labeled water. Journal of Applied Physiology, 81(2), pp.1019-1026.

Chan, C.B., Ryan, D.A.J. \& Tudor-Locke, C., 2004. Health benefits of a pedometer-based physical activity intervention in sedentary workers. Preventive Medicine, 39(6), pp.1215-1222.

Cocker, K.A.D., Bourdeaudhuij, I.M.D. \& Cardon, G.M., 2008. The effect of pedometer use in combination with cognitive and behavioral support materials to promote physical activity. Patient Education and Counseling, 70(2), pp.209-214.

Dinger, M.K. et al., 2007. Comparison of two emaildelivered, pedometer-based interventions to promote walking among insufficiently active women. Journal of Science and Medicine in Sport, 10(5), pp.297-302.

Guthold, R. et al., 2008. Worldwide Variability in Physical Inactivity. A 51-Country Survey. American Journal of Preventive Medicine, 34(6), pp.486-494.

Hildebrandt, V.H., TNO - Meer bewegen op het werk, Available at: http://www.tno.nl/downloads\%5CTNOKvL\%20Folder\%20Meer\%20bewegen $\% 20$ op $\% 20$ het $\% 20$ werk.pdf.

Matthews, T., 2006. Designing and evaluating glanceable peripheral displays. In Proceedings of the Conference on Designing Interactive Systems: Processes, Practices, Methods, and Techniques, DIS. pp. 343345.

Matthews, T., Rattenbury, T. \& Carter, S., 2007. Defining, designing and evaluating peripheral displays: An analysis using activity theory. Human-Computer Interaction, 22(1-2), pp.221-261.

Nutbeam, D. \& Harris, E., 2004. Theory in a Nutshell: A Practical Guide to Health Promotion Theories METRC., McGraw-Hill Book Company Australia.
Quesenbery, W., 2003. The five dimensions of Usability. In Content \& complexity: information design in technical communication. Routledge, p. 368.

Rosenstock, I., 1966. Why people use health services. Milbank Memorial Fund Quarterly-Health and Society, 44(3), pp.94-127.

Schulman, D. \& Bickmore, T.W., 2009. Persuading users through counseling dialogue with a conversational agent. In Proceedings of the 4th Int. Conf. on Persuasive Technology. Persuasive '09. ACM.

Stone, D. et al., 2005. User Interface Design and Evaluation, Morgan Kaufmann. 